\title{
Intelligent Monitoring System Based on Optical Fiber Acoustic Emission Sensor and Its Application in Partial Discharge Diagnosis of Gas-insulated Switchgear
}

\author{
Shiqi Hou, ${ }^{1}$ Yongrui Qin, ${ }^{2}$ Jiaxin Gao, ${ }^{1}$ Fuyong Lyu, ${ }^{1}$ and Xuefeng $\mathrm{Li}^{1 *}$ \\ ${ }^{1}$ College of Electronic and Information Engineering, Tongji University, \\ 4800 Cao'an Highway, Shanghai 201804, P. R. China \\ ${ }^{2}$ Department of Computer Science and Software Engineering, Xi'an Jiaotong-Liverpool University, \\ 111 Ren'ai Road, Suzhou 215123, P. R. China
}

(Received July 6, 2020; accepted January 11, 2021)

Keywords: partial discharge, optical fiber AE sensor, polarization modulation, BP-ANN

Gas-insulated switchgear (GIS) is widely used in high-voltage power transmission systems. There has also been increasing demand for the real-time and online detection of faults in GIS equipment. In this study, a new type of optical fiber acoustic emission (AE) sensor based on the photoelastic effect and the polarization modulation method is proposed and fabricated. Partial discharge (PD)-induced AE signals of different defects were collected by this sensor and used for back-propagation artificial neural network (BP-ANN) training and recognition after data preprocessing and feature extraction. The results of the research show that a BP-ANN with selfadaptation and self-learning combined with the proposed sensor has good performance in the recognition and prediction of PD faults in GIS equipment, and the average accuracy of the test set reached $93.7 \%$. The detection technology for weak AE signals and the fault identification method reported in this study can provide a reference for online monitoring of GIS and other equipment, which will have appreciable economic value and social significance.

\section{Introduction}

Gas-insulated switchgear (GIS) has been widely used in high-voltage power transmission systems. ${ }^{(1)}$ Most insulation faults of GIS are caused by partial discharges (PDs), which are induced by small insulation defects. ${ }^{(2,3)}$ This phenomenon gradually deteriorates the insulation performance and eventually leads to serious breakdown faults. The early detection of PDinduced acoustic emission (AE) signals can reduce the incidence of serious accidents caused by the insulation faults of GIS. ${ }^{(4)}$ Current detection methods mainly include the ultrahigh frequency (UHF) method, pulse current method, chemical detection method, optical detection method, and AE detection method. ${ }^{(5-7)}$ However, because these methods also have limitations, developing a method with higher accuracy to detect PD faults in GIS with a sealed metal shell is still a very significant engineering problem.

*Corresponding author: e-mail: lixuefeng@tongji.edu.cn https://doi.org/10.18494/SAM.2021.2974 
Optical fiber sensors have the advantages of insulation, anti-electromagnetic interference, corrosion resistance, and ease of realizing long-distance signal transmission. On the basis of the signal modulation method, optical fiber sensors can be classified as phase modulation type, intensity modulation type, wavelength modulation type, polarization modulation type, etc. ${ }^{(8-14)}$ Among them, fiber Bragg grating sensors and Fabry-Perot optical fiber sensors, whose techniques are relatively mature, have been widely used in various fields such as industry, the military, and aerospace. However, these two types of sensor have technical limitations that restrict their further application. For instance, fiber Bragg grating sensors mostly use the demodulation method of wavelength scanning, which results in a slow response and makes these sensors unsuitable for high-frequency AE signal acquisition. Also, their temperature cross-sensitivity is poor, with separate temperature compensation required. Although FabryPerot optical fiber sensors have high sensitivity, high resolution, and high accuracy, they usually require micromachining and coating of the fiber. These reduce the mechanical strength of the sensor and the stability of systems, making them unsuitable for long-term work in an industrial production site with temperature fluctuation, vibration, and shock.

After analyzing features of PD faults and comparing existing detection methods, in this study, we designed an optical fiber AE sensor based on the photoelastic effect and polarization modulation method to acquire PD-induced AE signals. Then, the fabricated sensor was used to collect PD-induced AE signals of different defects for building and training a back-propagation artificial neural network (BP-ANN) for recognizing defects. The results show that the system proposed in this study has good anti-interference ability and can collect weak AE signals and accurately identify different types of faults, which is of great significance for the safety monitoring of GIS equipment in ultrahigh voltage (UHV) power transmission systems.

\section{Sensor Design}

\subsection{Theory of photoelastic effect}

The photoelastic effect, which was first developed by the Scottish physicist David Brewster, was mathematically established by the French physicist Wertheim. ${ }^{(15)}$ For optical materials, the relationship among the refractive indices along the principal axes can be expressed as

$$
\frac{x^{2}}{n_{1}^{2}}+\frac{y^{2}}{n_{2}^{2}}+\frac{z^{2}}{n_{3}^{2}}=1 \text {, }
$$

where $n_{i}$ is the principal refractive index in the $i$ th direction in the optical material.

In the mechanical analysis of materials, the stress state at any point can be described by a quadric surface equation as follows:

$$
\sigma_{1} x^{2}+\sigma_{2} y^{2}+\sigma_{3} z^{2}=1,
$$

where $\sigma_{i}$ is called the principal stress in the direction of the $i$ th principal axis. 
Isotropic optical materials become anisotropic under stress load conditions, and the optical principal axes of a material are determined by the directions of its stress principal axes; in other words, they are coaxial. Considering that the optical principal axes are coaxial with the stress principal axes, the two interfaces of the two surface equations and their concentric spherical equations are parallel to each other. The following can be derived:

$$
\left\{\begin{array}{l}
n_{1}-n_{0}=-C_{1} \sigma_{1}-C_{2}\left(\sigma_{2}+\sigma_{3}\right) \\
n_{2}-n_{0}=-C_{1} \sigma_{2}-C_{2}\left(\sigma_{1}+\sigma_{3}\right), \\
n_{3}-n_{0}=-C_{1} \sigma_{3}-C_{2}\left(\sigma_{1}+\sigma_{2}\right)
\end{array}\right.
$$

where $C_{1}$ and $C_{2}$ are the first and second photoelastic constants, respectively, and their values only depend on the physical properties of the material. For pure silica, the material of optical fibers, we have $C_{1}=6.5 \times 10^{-13} \mathrm{~m}^{2} / \mathrm{N}$ and $C_{2}=4.2 \times 10^{-12} \mathrm{~m}^{2} / \mathrm{N}$.

Equation (3) describes the specific distribution of the refractive index of optical materials under stress load conditions, which provides an important theoretical basis for the research of optical fiber AE sensors.

\subsection{Polarization modulation}

In this study, a polarization modulation sensor structure based on fundamental-mode polarization interference was designed. Its principle is that the signal to be measured can be transformed into interference light intensity of two polarization modes by separating the orthogonal modes from the fiber, rotating one of them, and then combining one with the other. This type of sensor simultaneously has high sensitivity, a wide frequency bandwidth, and a fast response.

The principle of the optical fiber sensor based on polarization modulation is shown in Fig. 1. The incident light generated by the laser light source is converted from partially polarized light to linear polarized light by the polarizer. The linear polarized light is injected into the sensor at an angle of $45^{\circ}$ to the slow axis of a polarization-maintaining fiber (PMF), giving the components of light in the fast and slow axes the same initial intensity and phase. These two orthogonal polarized lights independently transmit with different transmission constants in the

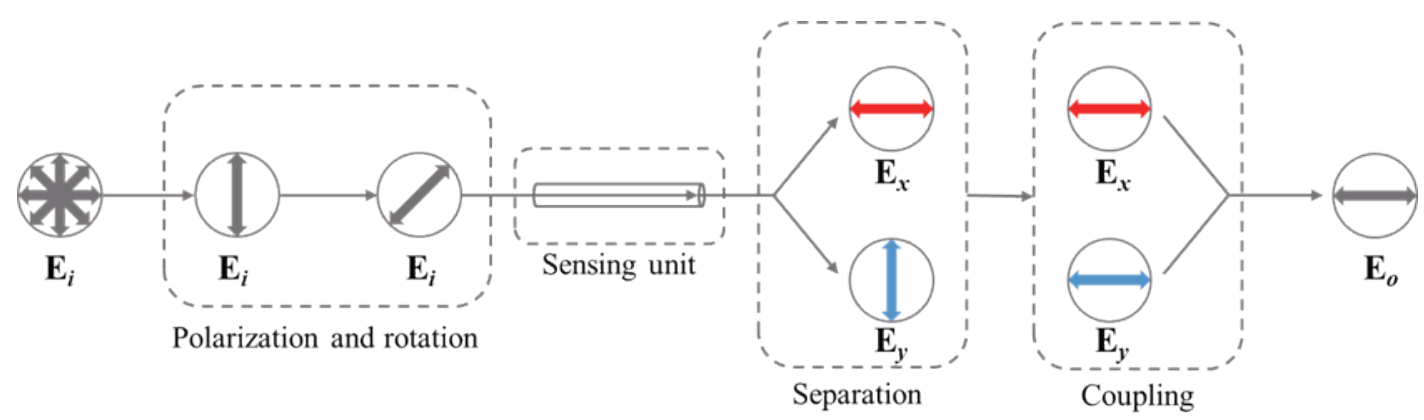

Fig. 1. (Color online) Schematic of the optical fiber sensor based on polarization modulation. 
fast and slow axes of the sensing unit, which causes a fixed phase difference at the output end. The two components are depolarized at the output end. Finally, the two depolarized components are superimposed to measure the interference light intensity to detect AE signals.

Assume that the fast and slow axes are in the $x$ and $y$ directions, respectively, with effective refractive indices $n_{x}$ and $n_{y}$, the initial electrical field of the linear polarized light is $E_{i}$, and the length of the fiber is $L$. Then, the output field is

$$
\left\{\begin{array}{l}
E_{x}=\frac{1}{2} E_{i} \cdot e^{-j \Phi_{x}} \\
E_{y}=\frac{1}{2} E_{i} \cdot e^{-j \Phi_{y}}
\end{array},\right.
$$

where $\Phi_{x}$ and $\Phi_{y}$ are the phases at the output end, which depend on the length of the optical fiber as the sensing unit, the wavelength of light, and the effective refractive index: $\Phi_{x}=2 \pi L \cdot n_{x} / \lambda=k_{0} n_{x} L, \Phi_{y}=2 \pi L \cdot n_{y} / \lambda=k_{0} n_{y} L$. The output light intensity can be described as

$$
I=\oint\left|E_{x}^{\prime}+E_{y}^{\prime}\right|^{2}=\left|E_{x}+E_{y}\right|^{2}=\frac{1}{4} E_{i}^{2} \cdot\left|e^{-j \Phi_{x}}+e^{-j \Phi_{y}}\right|^{2} .
$$

The phase property can be rewritten in trigonometric form and finally simplified to

$$
I=\frac{1}{2} E_{i}^{2} \cdot\left(1+\cos k_{0} L B\right) .
$$

Equation (6) describes the output light intensity of the polarization modulation sensor system. From Sect. 2.1, the distribution of the refractive index is affected by the external pressure load, leading to a change in birefringence. Therefore, the external pressure signal can be calculated from the change in the output light intensity.

When the optical fiber is not subject to external interference, the birefringence $B$ is a constant that depends on its optical property. It can be inferred from Eq. (6) that the output light intensity tends to change periodically with increasing fiber length $L$, with the period called the beat length of the fiber, which equals $\lambda / B$. For convenience, the total length of the fiber is assumed to be an integer multiple of the beat length, so that the output light intensity is maximum without pressure loading and that AE signals cause it to decrease.

As shown in Fig. 2, if we assume that AE signals affect the fiber on a part with length $l$, causing the effective refractive indexes in the fast and slow axis directions to change to $n_{x}^{\prime}$ and $n_{y}^{\prime}$, respectively, then the phase at the output end in Eq. (4) can be rewritten as

$$
\left\{\begin{array}{l}
\Phi_{x}=k_{0} n_{x}(L-l)+k_{0} n_{x}^{\prime} l \\
\Phi_{y}=k_{0} n_{y}(L-l)+k_{0} n_{y}^{\prime} l
\end{array} .\right.
$$




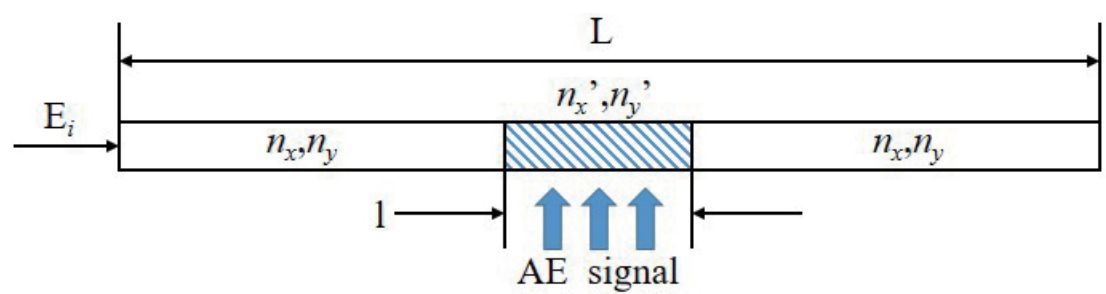

Fig. 2. (Color online) Influence of elastic wave on optical fiber properties.

Substituting Eq. (7) into Eq. (5), the phase property can be simplified to

$$
1+\cos \left[k_{0} B(L-l)+k_{0} B^{\prime} l\right]
$$

where $B^{\prime}=n_{x}^{\prime}-n_{y}^{\prime}$ is the birefringence of the part under the influence of the AE signal and $\Delta B=\left|B-B^{\prime}\right|$ is defined as the change in birefringence. The total length of the fiber is chosen as an integer multiple of the beat length. Then, after combining Eqs. (5) and (8), the output light intensity can be rewritten as

$$
I=\frac{1}{2} E_{i}^{2} \cdot\left(1+\cos \left(k_{0} l \Delta B\right)\right) .
$$

This equation describes the variation of the light intensity due to polarization modulation in the range of $l \Delta B<1 / \lambda$, and the variation is affected by both the influenced length $l$ and the change in birefringence $\Delta B$. In practical applications, the length $l$ can be controlled or fixed as a constant by adjusting the fiber-laying method, so the output light intensity only depends on the changes in birefringence. Therefore, the AE signal can be captured by real-time monitoring of the output light intensity; then, the characteristics of the AE signal can be extracted by time and frequency domain analyses.

\section{Data Acquisition and Processing}

Among the main causes of GIS faults, corona discharge between needle-plate electrodes in the loop circuit is the most common one. Therefore, we further analyzed this type of fault of PD. A model based on the above theory was used to simulate the generation of PD-induced AE signals at a voltage of $9 \mathrm{kV}$ in this study. Photographs of the sensor structure and the platform in the experiment are shown in Fig. 3. The experiment was divided into six groups according to the type of defects, and an optical fiber AE sensor (SM15-PS-U25D PANDA, Japan Fujikura Ltd.) was used to collect PD-induced AE signals generated by needle-plate electrodes set to electrode distances of 1 to $6 \mathrm{~mm}$. The collection time of each group was $200 \mathrm{~s}$, which was divided into 200 signals with an interval of $1 \mathrm{~s}$; therefore, we obtained a total of 1200 signals.

Owing to the influence of ambient noises during the AE signal acquisition, the signals were of poor quality as shown in Fig. 4(a). Thus, the signals and associated noises were preprocessed 


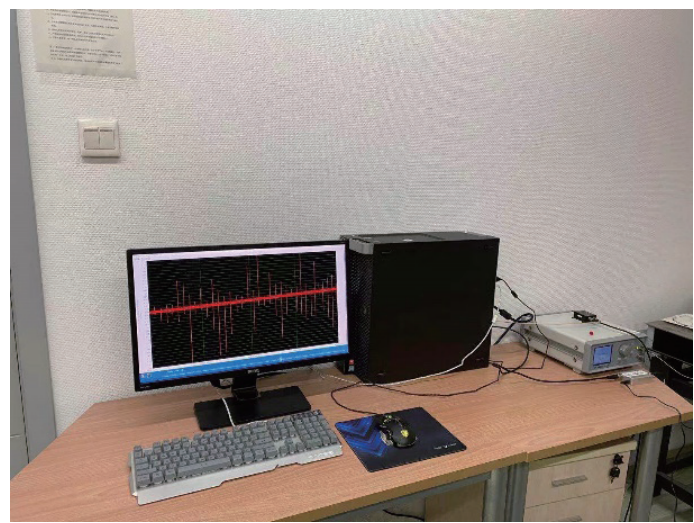

(a)

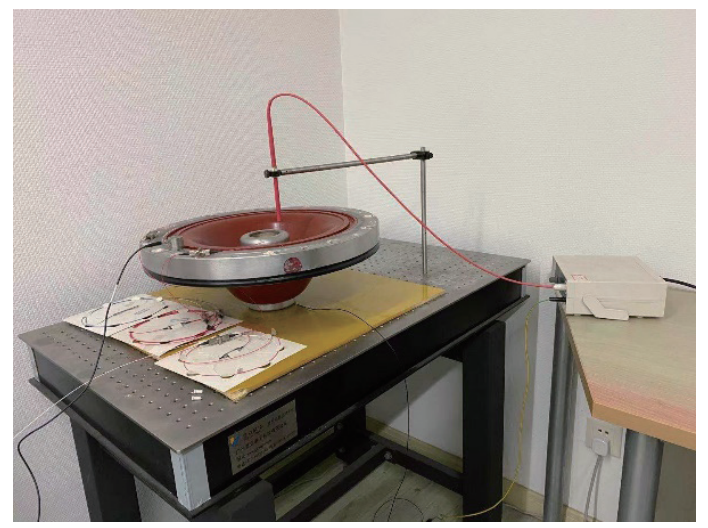

(b)

Fig. 3. (Color online) Photographs of the sensor structure and the platform in the experiment. (a) Data collection platform, (b) discharge platform.

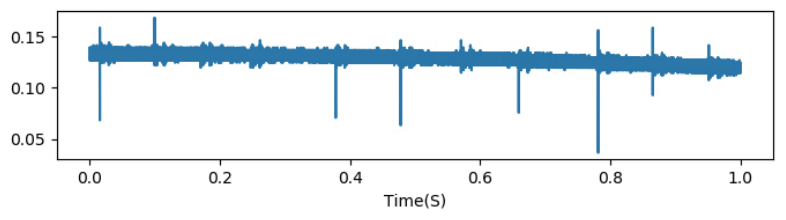

(a)

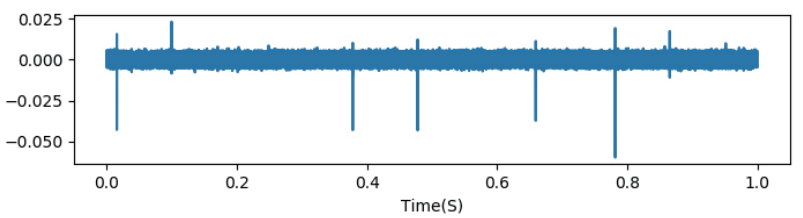

(c)

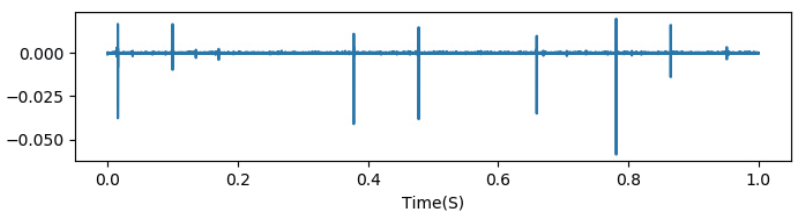

(e)

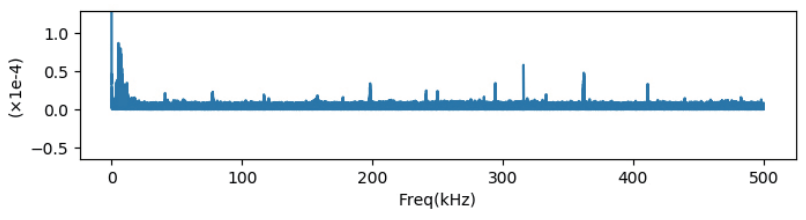

(b)

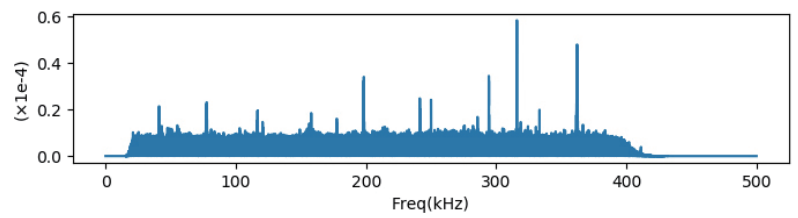

(d)

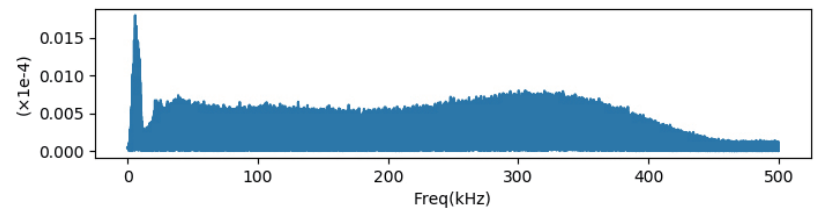

(f)

Fig. 4. (Color online) Time and frequency domain diagrams of the signal generated by $6 \mathrm{~mm}$ needle-plate electrodes. (a) Time domain diagram of the original signal, (b) frequency domain diagram of the original signal, (c) time domain diagram of the signal after filtering, (d) frequency domain diagram of the signal after filtering, (e) time domain diagram of the signal after filtering and denoising, and (f) frequency domain diagram of the signal after filtering and denoising.

before defect recognition. As shown in Fig. 4(b), the PD-induced AE signals were mainly distributed in the frequency range of $20-400 \mathrm{kHz}$, so the signals were first filtered by an appropriate bandpass filter. 
Figures 4(c) and 4(d) show that the noise was still strong compared with the AE signals after filtering the signals with the bandpass filter. The reason for this phenomenon is that the PD sources were inside the GIS equipment, which greatly attenuates the propagation of PD-induced AE signals. Thus, the signals with low SNR will decrease the defect recognition accuracy. Generally, AE signals behave as singular points, whose energy is concentrated on a few sparse wavelet coefficients with relatively large amplitudes. After an orthogonal wavelet transform, these coefficients are modal maximum values at various scales, while the stationary white noise is still white noise. ${ }^{(16)}$ Therefore, wavelet threshold denoising was used for further denoising, the results of which are shown in Figs. 4(e) and 4(f). It can be seen from the time domain diagram that signals with singularities and mutations were well preserved and that the noise was greatly reduced. Furthermore, it can be seen from Fig. 4(f) that the signal spectrum distribution had a large difference after noise reduction and exhibited many low-frequency components. They are considered to be generated by partial deformation of the signal during the decomposition and reconstruction. However, the energy source is still in the frequency range of $20-400 \mathrm{kHz}$, which must not be affected by low-frequency noise from the environment in the subsequent process of recognition and prediction.

\section{BP-ANN Defect Recognition Model}

In this study, a BP-ANN was chosen to construct the defect recognition model because of its advantages of nonlinearity, adaptability, and induction. Here, the sampling frequency of PDinduced AE signals was 1.0 MHz. Then, seven time domain features and 18 frequency domain features were extracted and respectively standardized in the time and frequency domains as shown in Table $1 .{ }^{(17-21)}$ Finally, the selected features were used to train a three-layer BP-ANN to construct a defect recognition model as shown in Fig. 5.

As previously mentioned, the data set had a capacity of 1200 and was divided into the training set and test set with a 3:1 ratio. After repeated experiments and parameter adjustments, the error of the model gradually converged to less than $10^{-2}$. Using the optimal parameters, the BP-ANN was randomly trained 10 times, and the recognition accuracy of the test set is shown in Fig. 6 . The average recognition accuracy of the test set reached $93.7 \%$ and the variance was 0.68 .

Table 1

List of the extracted features.

\begin{tabular}{ccc}
\hline & Time domain & Frequency domain \\
\hline Feature name & $\begin{array}{c}\text { Maximum value, minimum value, } \\
\text { absolute mean value, variance, standard } \\
\text { deviation, peak value, peak-to-peak value }\end{array}$ & $\begin{array}{c}\text { Maximum value of power spectrum, } \\
\text { median frequency, }\end{array}$ \\
\hline
\end{tabular}

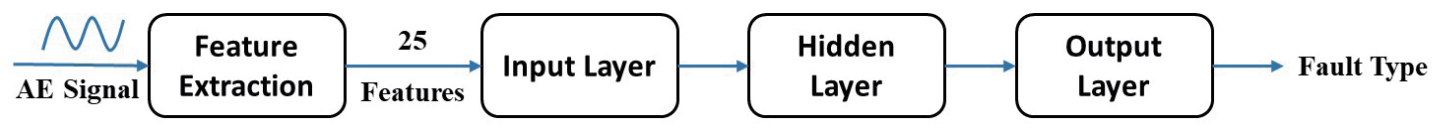

Fig. 5. (Color online) Structure diagram of the recognition model. 


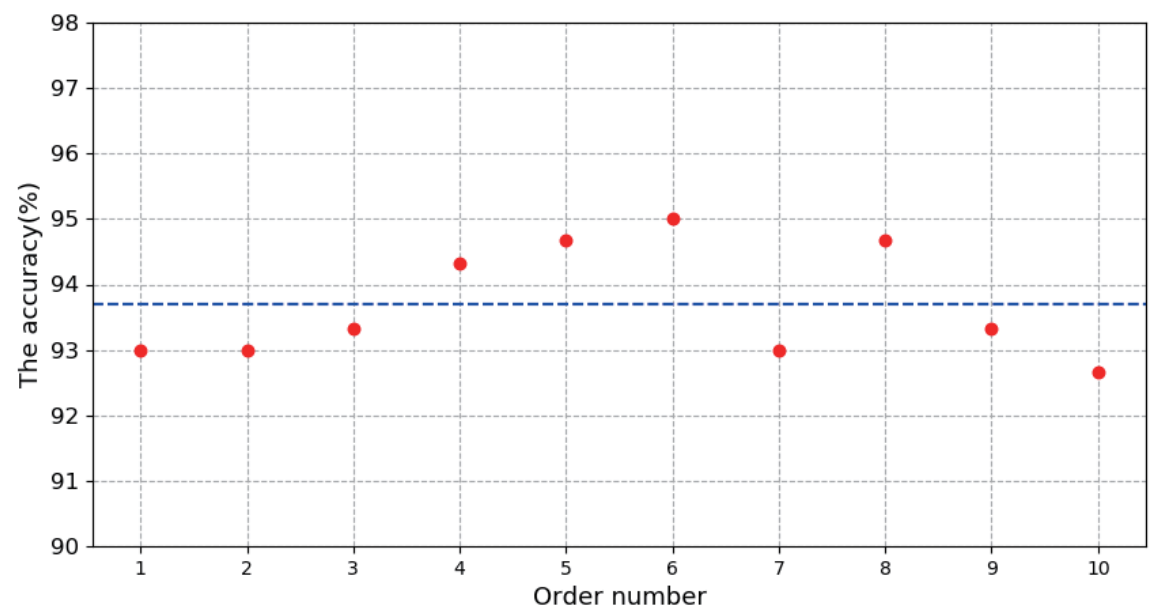

Fig. 6. (Color online) Recognition performance of proposed sensor on randomly selected test sets. The average accuracy was $93.7 \%$ and the variance was 0.68 .

The highest and lowest recognition accuracies of the test set were 95 and $92.7 \%$, respectively. The results show the validity of the data processing method and the model of PD-induced AE signal recognition, and verify the applicability of the proposed optical fiber AE sensor to PDinduced AE signal acquisition.

\section{Conclusions}

In this study, a new type of optical fiber AE sensor based on the principle of polarization modulation was proposed, which was used to collect PD-induced AE signals inside GIS equipment. The signals were preprocessed with bandpass filtering and wavelet threshold denoising because the original signals were doped with many low-frequency components and a large amount of noise. After that, feature extraction was performed on the signals in the time domain, frequency domain, and time-frequency domain, and the extracted features were used to train a BP-ANN to obtain a model that can accurately identify the PD phenomena of different defects. Results show that this optical fiber AE sensor combined with the trained BPANN defect recognition model can accurately predict the state of the internal fault source of fully enclosed metal GIS equipment by collecting, analyzing, and identifying PD-induced AE signals. The results of this research can enable online monitoring of PD phenomena from GIS equipment. The employed principle can also be used to detect PD phenomena in other scenarios, giving it universal significance as a reference for the intelligent health management of power equipment. However, this work also has some gaps and areas for improvement, for example, the experimental models were of a single type and the number of samples was relatively small. In future research, the operation data of production equipment in power enterprises will be collected for analysis and research to accelerate the application and development of this technology. 


\section{Acknowledgments}

This work was supported by Fundamental Research Funds for the Central Universities (No. 22120180189 and 22120190009) and National Natural Science Foundation of China (No. 61873189).

\section{References}

1 F. Wang and Y. Qiu: Power Syst. Technol. 27 (2003) 54. https://doi.org/10.13335/j.1000-3673.pst.2003.02.014

2 C. Gao, L. Yu, Y. Xu, W. Wang, S. Wang, and P. Wang: IEEE Trans. Power Delivery 1 (2018) 1. https://doi. org/10.1109/TPWRD.2018.2880230

3 W. Zhang, H. Zhao, and X. Chu: Acta Optic. Sin. 35 (2015) 57. https://doi.org/10.3788/AOS201535.0406002

4 B. Qi, C. Li, Z. Hao, and B. Geng: 2010 IEEE Int. Symp. Electrical Insulation (IEEE, 2010). https://doi. org/10.1109/ELINSL.2010.5549553

5 M. Ren, B. Song, T. Zhuang, and S. Yang: ISA Trans. 75 (2018) 247. https://doi.org/10.1016/j.isatra.2018.02.008

6 G. Ma, H. Zhou, C. Shi, Y. Li, and Q. Zhang: IEEE Sens. J. 18 (2018) 2788. https://doi.org/10.1109/ JSEN.2018.2803056

7 H. Zhou, G. Ma, M. Zhang, H. Zhang, and C. Li: IEEE Sens. J. 21 (2019) 24. https://doi.org/10.1109/ jsen.2019.2951613

8 J. Zhou, Y. Wang, C. Liao, B. Sun, J. He, G. Yin, S. Liu, Z. Li, G. Wang, X. Zhong, and J. Zhao: Sens. Actuators, B 208 (2015) 315. https://doi.org/10.1016/j.snb.2014.11.014

9 A. Rifat, R. Ahmed, A. Yetisen, H. Butt, and A. Sabouri, G. Mahdiraji, S. Yun and F. Adikan: Sens. Actuators, B 243 (2017) 311. https://doi.org/10.1016/j.snb.2016.11.113

10 B. Wu, C. Zhao, B. Xu, and Y. Li: Sens. Actuators, B 255 (2018) 3011. https://doi.org/10.1016/j.snb.2017.09.124

11 A. Socorro-Leránoz, D. Santano, D. Villar, and I. Matias: Biosens. Bioelectron.: X 1 (2019) 1. https://doi. org/10.1016/j.biosx.2019.100015

12 M. Zahid, J. Jiang, and S. Rizvi: Front. Optoelectron. 12 (2019) 215. https://doi.org/10.1007/s12200-019-0824-6

13 Z. Xu and X. Shu: J. Lightwave Technol. 37 (2019) 3503. https://doi.org/10.1109/JLT.2019.2917397

14 Y. Si, J. Lao, X. Zhang, Y. Liu, S. Cai, A. González-Vila, K. Li, Y. Huang, Y. Yuan, and C. Caucheteur: J. LightWave Technol. 37 (2019) 3495. https://doi.org/10.1109/JLT.2019.2917329

15 B. Zhang: J. Gansu Lianhe Univ. (Natural Science Edition) 27 (2013) 26. https://doi.org/10.3969/j.issn.1672691X.2013.05.008

16 Q. Wu and R. Zhang: Acta Optic. Sin. 40 (2020) 1. https://doi.org/10.3788/AOS202040.0810001

17 Q. Zhou, Y. Liu, J. Tang, and Y. Xie: 2008 Int. Conf. High Voltage Engineering and Application (ICHVE, 2008). https://doi.org/10.1109/ICHVE.2008.4774031

18 J. Tang, F. Liu, Q. Meng, X. Zhang, and J. Tao: IEEE Trans. Dielectr. Electr. Insul. 19 (2012) 37. https://doi. org/10.1109/TDEI.2012.6148500

19 W. Raymond, H. Lllias, A. Bakar, and H. Mokhlis: Measurement 68 (2015) 164. https://oi.org/10.1016/ j.measurement.2015.02.032

20 J. Tang, D. Wang, L. Fan, R. Zhuo, and X. Zhang: IEEE Trans. Dielectr. Electr. Insul. 22 (2015) 3037. https:// doi.org/10.1109/TDEI.2015.004556

21 X. Li, X. Wang, D. Xie, X. Wang, A. Yang, and M. Rong: IET Sci. Meas. Technol. 12 (2017) 169. https://doi. org/10.1049/iet-smt.2017.0287

22 D. Evagorou, A. Kyprianou, P. Lewin, A. Stavrou, V. Efthymiou, A. Metaxas, and G. Georghiou: IET Sci. Meas. Technol. 4 (2010) 177. https://doi.org/10.1049/iet-smt.2009.0023

23 R. Khushaba, S. Kodagoda, S. Lal, and G. Dissanayake: IEEE Trans. Biomed. Eng. 58 (2011) 121. https://doi. org/10.1109/TBME.2010.2077291

24 D. Wang, D. Miao, and C. Xie: Expert Syst. Appl. 38 (2011) 14314. https://doi.org/10.1016/j.eswa.2011.05.096 


\section{About the Authors}

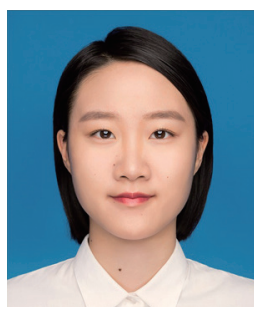

Shiqi Hou received her B.S. degree from Xiamen University, China, in 2019. Since 2019, she has been studying at Tongji University for her M.S. degree. Her research interests are in optical fiber sensors, signal processing, and mathematical modeling. (1930730@tongji.edu.cn)

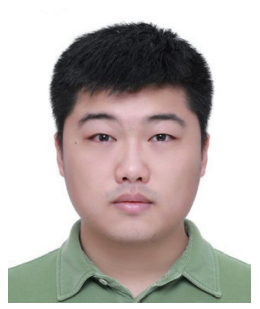

Yongrui Qin has been studying at Xi'an Jiaotong-Liverpool University for his Bachelor of Arts in Computer Science since 2016. His research interests are in programming and artificial intelligence.

(Yongrui.Qin16@student.xjtlu.edu.cn)

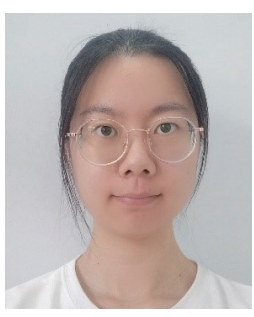

Jiaxin Gao has been studying at Tongji University for her B.S. degree since 2017. Her research interests are in controlling and modeling.

(1752276@tongji.edu.cn)

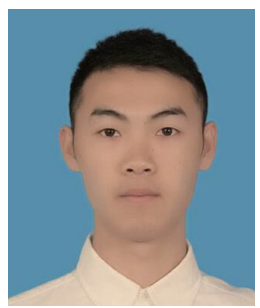

Fuyong Lyu received his B.S. degree from Tongji University in 2019 and has been pursuing his M.S. degree since 2019. He is mainly engaged in research on optical fiber sensor development, detection technology, and automation devices. (1932972@tongji.edu.cn)

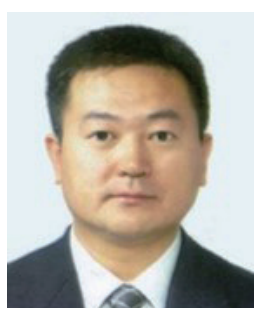

Xuefeng Li graduated from Shenyang Institute of Engineering, China, in 1999. He received his M.E. and D.E. degrees from Fukuoka Institute of Technology, Fukuoka, Japan, in 2004 and 2007, respectively. From 2007 to 2013, he was a postdoctoral and assistant researcher at Waseda University, Kitakyushu, Japan. Since 2010, he has been an associate professor at Tongji University. His research interests are in sensors and nondestructive testing. (lixuefeng@tongji.edu.cn) 